\title{
How Scripps Networks uses digital asset management to market its inventory of television programs to advertisers
}

\author{
Karen Fitchpatrick \\ is Director of Media Applications, Scripps Networks. She has over 15 years of experience in IT management and development, working \\ with companies such as MGM, Spelling Entertainment, Paramount and Scripps Networks to define and implement digital asset \\ management (DAM) strategies. In support of these strategies she provides a strong financial background which drives business case \\ development and financial management.
}

Keywords: publishing, advertising, cable television, end-user, advertisers, broadband

Abstract Scripps Networks was faced with a common issue: how to best leverage available inventory across all distribution channels. As the owner of four successful cable channels, as well as award-winning websites, Scripps has an extensive library of content from which to generate an ad inventory. The key is how to determine the best mix of inventory across the disciplines in order to maximize revenues. The distribution channels at Scripps include cable, online, video on demand (VOD) and broadband. In order to maximize the potential for the network as a whole, the ad sales team needs a view of available inventory across all distribution channels as well as the specific programming around that inventory. In addition, the sheer volume of ads in their various formats requires an enterprise-level system to track and manage them. To that end, we are working towards an integrated solution that provides a unified view across the disciplines. The software applications that make up the digital asset management (DAM) solution at Scripps Networks include not only the traditional asset management software but also sales, traffic, campaign management, billing and reporting. Our asset management solution includes both content assets and advertising assets for distribution across on-air, online, VOD and broadband. The rationale is that for Scripps, our content is the asset and without cross-discipline management tools, we cannot effectively and efficiently manage the content and advertising opportunities.

Email: kfitchpatrick@

scrippsnetwork.com
Scripps Networks, the parent company of the popular lifestyle television brands Home \& Garden Television (HGTV), Food Network, DIY Network and FINE LIVING, as well as electronic retailer, Shop At Home, and music network, Great American Country, was faced with a common issue among content providers: how best to leverage available inventory across all of the company's content platforms. Scripps has an extensive library of content from which to generate ad inventory. The key is how to determine the best mix of inventory across the disciplines in order to maximize revenues. In order to accomplish this, senior management needs to view inventory across all the distribution channels.
At Scripps, the content platforms include cable/satellite television, online, video on demand (VOD) and syndicated broadband. Cable and satellite refers to the traditional television networks supported by advertising and affiliate sales. Online is the web presence that complements the on-air programming (HGTV.com, FoodNetwork.com, DIYnetwork.com and FineLiving.com) that provide audiences with show references, detailed project instructions, materials lists and recipes corresponding to the television networks.

VOD is long-form and short-form programming that utilizes library content and repackages it to provide new and compelling content to viewers. On the technical side, VOD 
consists of MPEG 3.x video distributed by the cable operators. Broadband is delivered over the internet via third-party internet partners such as MSN and Comcast.Net, but is differentiated from online resource by the emphasis on high bandwidth content, particularly video.

Advertising opportunities are different for each discipline. Cable consists of a predetermined amount of advertising approximately eight minutes for each 30 minutes on the air, governed by federal regulations. Advertising during certain dayparts carries a premium over others and requires that we maximize every second of inventory available during those peak times.

Online resource has much more flexible availability not only in terms of volume, but also by types of ads. There are rich media ads, video ads (traditional commercials), and static graphical ads. The myriad of available combinations provides more flexibility for advertisers by offering more targeted ad campaigns than traditional methods.

Advertising for VOD is somewhat problematic because the current environment requires that the ads be embedded in the MPEG stream so content and ads can be delivered seamlessly. The industry is changing to allow dynamic ad insertion which will more closely align VOD with the traditional cable advertising. However, this synchronization is approximately 12-18 months away.

Broadband combines the flexibility and targeting capabilities of online resource with the high quality video of on-air. The broadband ad market is just getting off the ground but is recognized by advertisers and content providers as a golden opportunity for reaching viewers.

In order to maximize the potential for Scripps Networks as a whole, the ad sales team needs a view of available inventory across all distribution platforms as well as the specific programming around that inventory. In addition, the sheer volume of ads in their various formats requires an enterprise-level system to track and manage them. To that end, we are working towards an integrated solution that provides a unified view across the disciplines.

The end user experience occurs when the content and advertising come together. The way this happens is different for each content type, as each is at a different point in its lifecycle.
Cable goes on-air by automated applications that are directed by a traffic log. The traffic log is generated by combining segment data from the content episodes and a program schedule with commercials and other advertising elements from the advertising sales process. The logs are created automatically based on business rules and then manipulated manually to fill in any gaps.

Online resource is presented to the user by inserting links in a web page that call the online ad traffic application which interprets the "call" and displays the appropriate advertizing based on the current campaign and business rules. The ads can be multiple formats and can be tied to each other through the rules. For example, a Flash ad and a graphic ad for the same advertiser can be displayed on a specific page according to the ad campaign manager.

VOD is trafficked similar to on-air based on business rules and the advertising campaign and is then edited together into a single MPEG stream for delivery to the cable operators. The lead time for VOD is significantly higher than cable because of the requirement to deliver a single file. The advertising is embedded in the programming during the editing process, which occurs several weeks in advance of distribution.

Broadband is similar to online resource but provides the opportunity to present video ads in a video content stream similar to the on-air model. A playlist is generated like the traffic $\log$ that identified content segments and advertising and determines when they are delivered to the user and in what order. In addition, the traffic application can serve graphic ads that are coordinated with the video ad based on business rules.

The four distribution channels include many types of assets from content to ads, videos to graphics, MPEGs to JPEGs. As you might imagine, the management of all these assets is a monumental task. The challenge for our software applications is to deliver content and advertising as close to the user "experience" as possible, thus providing opportunities for dynamic ad insertion across all distribution channels while maximizing the advertising potential.

The software applications that make up the DAM solution at Scripps Networks include not only the traditional asset management software 
but also sales, traffic, campaign management, billing and reporting. Our asset management solution includes both content assets and advertising assets for distribution across on-air, online, VOD and broadband. The rationale is that for Scripps, our content is the asset and without cross-discipline management tools, we cannot effectively and efficiently manage the content and advertising opportunities.

We have defined a roadmap which gets us to the point where we will have a unified view of our content and advertising and be able to maximize our assets and our advertising inventory. The roadmap allows for ultimate scalability and flexibility to support increased volumes and new distribution channels. The current roadmap started to take shape three years ago. Scripps Networks defined an enterprise roadmap for asset management based on the overall business plan drivers including increasing brand viewership, improving operational efficiency, expanding revenue opportunities and improving content accessibility. We aligned the phased roadmap to the business drivers which enables us to extend the roadmap to new ventures more easily. For example, because of the way the roadmap had been implemented for improving content accessibility, we were able to extend that to broadband when it became "hot" last year. We were up and running with a new distribution channel in just a few months.

It is important to keep the roadmap current which is why we review our position every six months or so and update the map with revised timelines and applications. We are currently executing an extensive strategic planning exercise to confirm the existing business drivers and reconfirm the roadmap to look ahead for the next 18-24 months, particularly in the new emerging media market segments. The original roadmap was closely aligned to the existing business drivers but consistent review and alignment towards new and future distribution channels will enable Scripps Networks to address technology needs before they are required for actual business implementation.

Specific to how we use asset management in marketing inventory of television programs to advertisers - during our original roadmap design, we determined that much of our mission critical data was in more than 100 Filemaker Pro databases. To achieve an enterprise-wide view of inventory, we mapped out a plan to consolidate the multiple legacy applications into a handful of integrated enterprise applications. These included Webware Active Media, CAM's Broadway suite, Nesbit Media Library System and some custom-developed applications for content tracking. We engaged some key development partners such as Ascertane to ensure critical components were implemented correctly and we also leveraged some critical relationships with third-party vendors, including thePlatform, to assist in distributing our content. These vendors, tied closely with our internal development and project management teams, have enabled us to deploy enterprise applications to replace the "silo'd" Filemaker databases which had sprung up on their own.

With this many vendors involved in our critical applications, vendor management becomes extremely important. We accomplished this by defining master terms for each vendor and assigning an internal project manager to manage the day-to-day relationships. Our project management team is comprised of individual managers who focus on specific business areas, including cable distribution, emerging media opportunities and legacy application conversion.

A key component of our application consolidation is the integration of the applications. As we work towards a unified view of assets and available inventory, it becomes critical that the various applications supporting these areas can "talk" to each other effectively. The ideal flow of information is:

1 A sales rep puts together a plan that includes cable, online, VOD and broadband advertising opportunities. The plan recognizes the available inventory across all content platforms and confirms availability for the rep. The plan should be entered into one system which assimilates the information and determines which subsystem should handle which piece of the proposal.

2. The planning system parses out the information to the systems that support each of the platforms so the appropriate trafficking information can be associated with each order.

3. The designated traffic system assigns the 
appropriate advertising with the appropriate content based on campaign management and business rules.

4. The various traffic systems then send the appropriate information to the consolidated billing system for reconciliation and invoicing.

Ultimately, asset management is critical in
Scripps' management of content and advertising and bringing the two together for the end user in whatever medium the user chooses. Whether it is traditional television viewing, VOD, traditional website properties or the newest syndicated broadband systems, without DAM, Scripps would not be able to provide advertisers with the critical information they need to make the advertising buy. 